\title{
Successful treatment of allergic bronchopulmonary aspergillosis with recombinant anti-lgE antibody
}

\author{
Cornelis K van der Ent, Hans Hoekstra, Ger T Rijkers
}

Thorax 2007;62:276-277. doi: 10.1136/thx.2004.035519

Allergic bronchopulmonary aspergillosis (ABPA) can cause severe worsening of the respiratory condition in patients with cystic fibrosis. Treatment can result in steroid dependency and serious adverse events. A dramatic and rapid improvement of respiratory symptoms and lung function after a single dose of anti-lgE antibody (omalizumab) in a 12-year-old girl with cystic fibrosis and ABPA is described. This is the first report of this experimental treatment. It suggests an important role for $\lg E$ in the pathogenesis of ABPA and offers new therapeutic possibilities.

\begin{abstract}
$\Lambda$ llergic bronchopulmonary aspergillosis (ABPA) occurs in up to $15 \%$ of patients with cystic fibrosis. ${ }^{1}$ Clinically, the disease is manifested by wheezing, pulmonary infiltrates and bronchiectasis. A number of immunological responses to antigens of Aspergillus fumigatus can be observed, such as peripheral blood eosinophilia, immediate cutaneous reactivity, increased levels of total serum IgE, the presence of precipitating antibodies and increased specific serum IgE and IgG antibodies to A fumigatus. ${ }^{1}$ Treatment for ABPA involves attenuation of the inflammatory and immunological activity with corticosteroids and reduction of the antigen burden from fungal colonisation with antifungal agents. Long-term treatment is often required because of exacerbations that repeatedly occur when corticosteroid treatment has been tapered. Serious adverse events to corticosteroids are frequent in these patients. In this report, we successfully treated a patient with recombinant anti-IgE antibody (omalizumab).
\end{abstract}

\section{CASE HISTORY}

A 12-year-old girl had been diagnosed with cystic fibrosis directly after birth because of meconium ileum (genotype dF508/dF508). From infancy she had mild to moderate lung problems requiring antibiotic treatment three to five times a year. From age 5 years she was colonised with Pseudomonas aeruginosa and A fumigatus. At age 8 years she had more severe signs of cough and exercise-induced asthma. She developed pulmonary infiltrates and bronchiectasis and signs of severe airflow obstruction (forced expiratory volume in $1 \mathrm{~s}\left(\mathrm{FEV}_{1}\right)$ fell from $105 \%$ to $34 \%$ of predicted). Blood testing revealed eosinophilia, marked increase in total serum IgE level (5200 IU/ml), positive Aspergillus-specific IgE-radio allergosorbent test (class 4-5) and positive precipitating IgG antibodies to Aspergillus. The diagnosis of ABPA was made and corticosteroids were started (prednisone $50 \mathrm{mg} /$ day). Within several days, her symptoms disappeared and the lung function parameters returned to normal. The corticosteroids could be tapered after 3 weeks. Between ages 8 and 12 years, exacerbations of ABPA recurred many times when the dosage of prednisone was reduced below 10-15 mg/day. Prednisone could not be stopped. At 12 years of age, she developed the complete spectrum of severe corticosteroid-related adverse events (growth retardation, adiposity, hypertension and osteoporosis). For this reason, we considered an alternative treatment strategy-namely, treatment with omalizumab. After cessation of the corticosteroids her $\mathrm{FEV}_{1}$ fell from $105 \%$ to $65 \%$ of predicted in 2.5 weeks time (fig 1). Subcutaneous administration of a single dose of $300 \mathrm{mg}$ omalizumab resulted in complete disappearance of complaints of dyspnoea and an increase in $\mathrm{FEV}_{1}$ to $98 \%$ of predicted within $4 \mathrm{~h}$. After 2 weeks her lung function began to decline again. $\mathrm{FEV}_{1}$ was $64 \%$ of predicted 18 days after the first dose, and a second dose of omalizumab was given. The same clinical results were observed repeatedly in the period thereafter (fig 1 ). To date, the patient is free of corticosteroids and doing well clinically. During the treatment period no changes in total or specific immunoglobulins (IgE, IgG and IgA) were observed. However, omalizumab can interfere with accurate quantitation of serum IgE levels as a result of omalizumab-IgE binding. During treatment no clinical detectable adverse effects were observed.

\section{DISCUSSION}

To our knowledge, this is the first patient with cystic fibrosis with ABPA to be treated with omalizumab. There was a repeated improvement in symptoms and normalisation of lung function within $2-4 \mathrm{~h}$ of administration of the drug. Several lessons can be drawn from this observation.

Omalizumab improves asthma control in patients with severe allergic asthma, reducing inhaled corticosteroid requirements. ${ }^{2}$ Although in patients with asthma the exacerbation rate declines during treatment, the effects on lung function remain relatively small. The dramatic improvement in both respiratory symptoms and $\mathrm{FEV}_{1}$ in our patient suggests that omalizumab might offer a new therapeutic approach to patients with ABPA. Of course, our unblinded observations are not ultimate proof of the efficacy of omalizumab in ABPA. However, the striking, consistent and repeatable effects of subsequent administrations

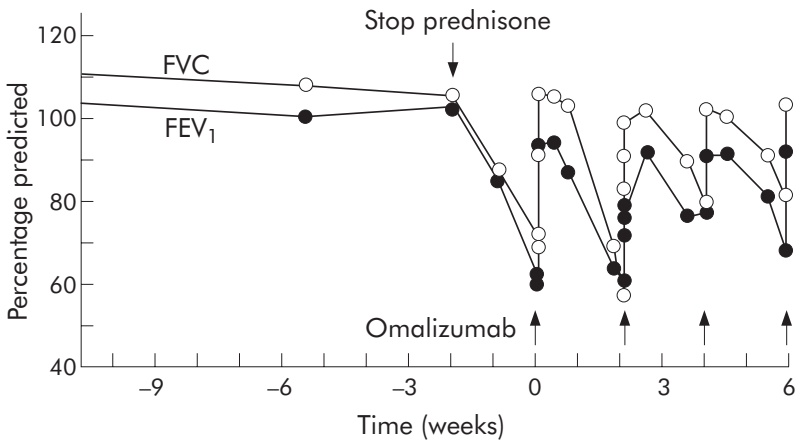

Figure 1 Lung function parameters (forced vital capacity (FVC) and forced expiratory volume in $1 \mathrm{~s}\left(\mathrm{FEV}_{1}\right)$, expressed as percentages of predicted) before and after treatment with omalizumab. 
indicate effectiveness in our patient and justify future randomised clinical trials.

The pathogenesis of ABPA remains complex and confusing. In all patients, very high total serum IgE levels and high levels of IgE anti-Aspergillus antibodies are seen. There seem to be quantitative and perhaps qualitative differences in the B cell IgE antibody responses between those with ABPA and those without ABPA. ${ }^{3}$ In ABPA, there are also increased amounts of IgG and IgA anti-Aspergillus antibodies, which reflect the balance between $\mathrm{T}$ helper cells types 2 and 1 in the response to Aspergillus antigens in these patients. ${ }^{4}$ To date, it has not been clear whether extreme amounts of IgE play a role in the pathogenesis of ABPA or whether it is only an epiphenomenon. The timing of the effects after administration of omalizumab in our patient suggests that IgE antibody might have an important role in the signs of airflow obstruction in ABPA. The serum levels of free $\operatorname{IgE}$ are reduced in a dose-dependent manner within $2 \mathrm{~h}$ of subcutaneous administration of omalizumab. The half-life of the drug in serum is about 22 days. ${ }^{5}$ In our patient, clinical improvement was observed within several hours, lasting for 2-3 weeks.

The diagnosis of ABPA in cystic fibrosis is difficult because many of the diagnostic criteria overlap with common manifestations of cystic fibrosis. The diagnosis is based on the presence of five or more essential criteria as proposed by the international consensus committees, ${ }^{1}$ and specific tests are lacking. When our observation is confirmed in other patients, the rapid and clear improvement of clinical signs and lung function after a single dose of omalizumab might be used as a helpful diagnostic test for ABPA.

\section{Authors' affiliations}

Cornelis K van der Ent, Department of Pediatric Respiratory Diseases, University Medical Center Utrecht, Utrecht, The Netherlands

Hans Hoekstra, Department of Pediatrics, Hieronymus Bosch Hospital, Hertogenbosch, The Netherlands

Ger T Rijkers, Department of Pediatric Immunology, University Medical Center Utrecht, Utrecht, The Netherlands

Funding: None.

Competing interests: None.

Correspondence to: $\mathrm{Dr} C \mathrm{~K}$ van der Ent, Department of Pediatric Respiratory Diseases, University Medical Center Utrecht, KH 01.419.0, PO Box 85090, 3508 AB Utrecht, The Netherlands; k.vanderent@wkz.azu.nl

Received 30 September 2004

Accepted 17 January 2005

\section{REFERENCES}

1 Stevens DA, Moss RB, Kurup VP, and participants in the Cystic Fibrosis Foundation Consensus Conference, et al. Allergic bronchopulmonary aspergillosis in cystic fibrosis-state of the art: Cystic Fibrosis Foundation Consensus Conference. Clin Infect Dis 2003;37(Suppl 3):S225-64.

2 Holgate ST, Chuchalin AG, Hebert J, et al. Efficacy and safety of a recombinant anti-immunoglobulin $\mathrm{E}$ antibody (omalizumab) in severe allergic asthma. Clin Exp Allergy 2004;34:632-8.

3 El Dahr JM, Fink R, Selden R, et al. Development of immune responses to Aspergillus at an early age in children with cystic fibrosis. Am J Respir Crit Care Med 1994;150:1513-18.

4 Leser C, Kaufmann HF, Virchow C Sr, et al. Specific serum immunopatterns in clinical phases of allergic bronchopulmonary aspergillosis. J Allergy Clin Immunol 1992:90:589-99.

5 Hochhaus G, Brookman L, Fox $\mathrm{H}$, et al. Pharmacodynamics of omalizumab: implications for optimised dosing strategies and clinical efficacy in the treatment of allergic asthma. Curr Med Res Opin 2003;19:491-8.

\section{LUNG ALERT}

Inducible bronchus-associated lymphoid tissue and the pathogenesis of rheumatoid lung

$\Delta$ Rangel-Moreno J, Hartson L, Navarro C, et al. Inducible bronchus-associated lymphoid tissue (iBALT) in patients with pulmonary complications of rheumatoid arthritis. J Clin Invest 2006;1 16:3183-94.

B ronchus-associated lymphoid tissue (BALT) comprises submucosal lymphoid cells analogous to Peyer's patches but located in association with large airways. It is generally not found in normal mouse or human lung tissue. Pulmonary infection or inflammation in mice leads to the development of lymphoid follicles that are not restricted to the upper airways, termed inducible BALT (iBALT). In this study the authors examined lung biopsies from patients with a variety of interstitial diseases including idiopathic pulmonary fibrosis, hypersensitivity pneumonitis and lung disease in association with rheumatoid arthritis (RA) and Sjogren's syndrome (SS). Although small amounts of lymphoid aggregates were found in the nonautoimmune conditions, samples from the patients with RA and SS had more and larger lymphoid areas.

The authors then proceeded to investigate the potential roles and causes of this well-organised iBALT. First, venules and lymphatic vessels were consistently found within the follicles and it was felt they were likely to facilitate entry and exit of immune cells. Second, levels of certain cytokines involved in lymphoid organogenesis and $\mathrm{T}_{\mathrm{H}} 2$-driven pathology, as well as levels of the autoantibody anti-cyclic citrullinated peptide (anti-CCP) in BAL fluid, correlated well with the amount of iBALT. Third, dense areas of collagen deposition and myofibroblast activity around the follicles were seen in the patients with highly organised iBALT.

These findings suggest that iBALT may have a significant role in the pathogenesis of interstitial lung disease seen in association with connective tissue disorders. Further understanding of the mechanisms involved could be used to develop novel treatments.

R Condliffe

Specialist Registrar, Royal Hallamshire Hospital, Sheffield, UK; condliffe@doctors.org.uk 